\title{
Decreased miRNA-148a is associated with lymph node metastasis and poor clinical outcomes and functions as a suppressor of tumor metastasis in non-small cell lung cancer
}

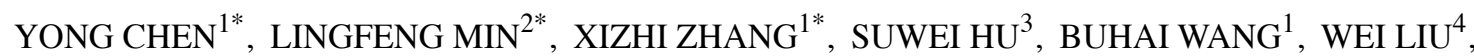 \\ RUI WANG ${ }^{5}$, XUEWEN GU ${ }^{6}$, WEIGAN SHEN ${ }^{7}$, HOUNING LV ${ }^{7}$, JING ZOU ${ }^{2}$, \\ YUEXIANG CHEN ${ }^{6}$, XINGXIANG $\mathrm{XU}^{2}$ and LONGBANG $\mathrm{CHEN}^{5}$
}

\begin{abstract}
Departments of ${ }^{1}$ Medical Oncology, ${ }^{2}$ Respiratory Medicine and ${ }^{3}$ Gynecology and Obstetrics, Subei People's Hospital, Clinical Medical College of Yangzhou University, Yangzhou, Jiangsu 225001; ${ }^{4}$ Department of Medical Oncology, Hebei Medical University Fourth Hospital, Shijiazhuang, Hebei 050011; ${ }^{5}$ Department of Medical Oncology, Jinling Hospital, School of Medicine, Nanjing University, Nanjing, Jiangsu 210002; ${ }^{6}$ Department of Pathology, Subei People's Hospital, Clinical Medical College of Yangzhou University, Yangzhou, Jiangsu 225001;

${ }^{7}$ Department of Cytobiology, School of Medicine, Yangzhou University, Yangzhou, Jiangsu 225001, P.R. China
\end{abstract}

Received May 20, 2013; Accepted June 21, 2013

DOI: $10.3892 / o r .2013 .2611$

\begin{abstract}
Non-small cell lung cancer (NSCLC) is the leading cause of cancer-related death worldwide, and only $15 \%$ of lung cancer patients live more than 5 years. microRNAs (miRNAs) are endogenously expressed non-coding RNAs, and dysregulation of miRNAs is a common feature in human cancers including lung cancer. In this study, we describe the epigenetic regulation of miRNA-148a and its prognostic value in NSCLC. Due to hypermethylation of the miRNA-148a encoding region, the expression levels of miRNA-148a were decreased in NSCLC tissues and cells. Decreased miRNA-148a expression was associated with lymph node metastasis, advanced clinical stage and shortened disease-free survival and overall survival in NSCLC, and was an independent prognostic factor for overall survival in multivariate analysis. In vitro, overexpression of miRNA-148a significantly suppressed the migratory and invasive abilities of A549 and H1299 lung cancer cells.
\end{abstract}

Correspondence to: Professor Longbang Chen, Department of Medical Oncology, Jinling Hospital, School of Medicine, Nanjing University, 140 Hanzhong Road, Nanjing, Jiangsu 210002, P.R. China

E-mail: dr.chenlb@163.com

Professor Xingxiang Xu, Department of Respiratory Medicine, Subei People's Hospital, Clinical Medical College of Yangzhou University, No. 98 Nantong West Road, Yangzhou, Jiangsu 225001, P.R. China

E-mail: xuxx63@sina.com

${ }^{*}$ Contributed equally

Key words: miRNA-148a, non-small cell lung cancer, methylation, clinicopathologic characteristics, prognostic value
Enforced expression of miRNA-148a in lung cancer cell lines resulted in a significant reduction in the expression of DNMT1. This, in turn, led to a decrease in DNA methylation of the tumor-suppressor gene E-cadherin and induced an increase in the protein levels of E-cadherin. By understanding the function and molecular mechanism of miRNA-148a in NSCLC, miRNA-148a may have therapeutic potential to suppress lung cancer metastasis.

\section{Introduction}

Lung cancer is the leading cause of cancer-related death worldwide including China (1). In most patients, non-small cell lung cancer (NSCLC), which accounts for $70-80 \%$ of lung cancer cases, is generally diagnosed at an advanced stage accompanied by extensive invasion or lymphatic metastasis. Successful clinical strategies are limited, and the prognosis of NSCLC patients is still very poor. Only $15 \%$ of lung cancer patients live more than 5 years after diagnosis. Lung carcinogenesis is a multistep process, which is currently believed to be associated with the activation of oncogenes and the inactivation of tumor-suppressor genes (2). To date, the molecular mechanisms underlying the progression of NSCLC are still poorly understood. Therefore, investigations into the molecular mechanisms involved in NSCLC development have major importance and may be helpful to develop novel therapeutic targets and strategies for the treatment of human NSCLC.

microRNAs (miRNAs) are endogenously expressed non-coding RNA molecules that cause mRNA translation inhibition or degradation via direct base-pairing interactions, which mainly repress the expression of several putative targets post-transcriptionally (3). With the development of high throughput technologies such as microarray platforms, more and more miRNAs have been found to be abnormally expressed in a variety of cancers $(4,5)$. Moreover, emerging 
evidence demonstrates that miRNAs are abnormally expressed in various types of cancers, and act as oncogenes or tumor-suppressor genes to control many fundamental cellular processes, including tumorigenesis, cellular differentiation, proliferation, apoptosis, tumor invasion, metastasis and therapeutic response $(6,7)$. Furthermore, altered expression of certain miRNAs can also serve as effective biomarkers for predicting prognosis in multiple tumor types (8-10). Lung cancer is associated with unique dysregulated miRNAs $(4,11)$; among them, miRNA-148a has been found to be one of the downregulated miRNAs (11).

Currently, there is a consensus that expression change of miRNA-148a is very important for cancer development. Studies have shown that miRNA-148a is downregulated in cancers, including chronic lymphocytic leukemia (12), hepatoblastoma (13), breast (14), gastrointestinal carcinoma (15-19) and pancreatic ductal adenocarcinoma (20). It has been shown to suppress tumor growth, angiogenesis, invasion and dissemination and promote apoptosis in different types of human tumors $(14,18,20,21)$. Furthermore, low levels of miRNA-148a were observed to be associated with advanced $\mathrm{T}$ stage, increased tumor size and lymph node metastasis in gastrointestinal cancers $(17,22)$. miRNA-148a was also negatively associated with tumor differentiation in adenocarcinoma of the esophagus (9). Yet, in NSCLC, there are no data available evaluating the association between miRNA-148a expression and clinicopathological features and clinical outcomes. Moreover the possible molecular mechanisms in NSCLC metastasis are still not well elucidated.

In this study, our data suggest that DNA methylation is involved in the downregulation of miRNA-148a in NSCLC. Further studies have demonstrated that decreased miRNA-148a expression is closely associated with lymph node metastasis, advanced clinical stage and poor survival, and is an independent prognostic factor for overall survival. Moreover, miRNA-148a induces demethylation of E-cadherin promoters and upregulates E-cadherin expression by repressing expression of DNA methyltransferase 1 (DNMT1), which in turn leads to suppression of the migratory and invasive abilities of lung cancer cells.

\section{Materials and methods}

Ethical approval of the study protocol. The present study was approved by the Ethics Committee of Subei Hospital (Jiangsu, China), and written informed consent was obtained from all patients.

Study population. Ten pairs of formalin-fixed, paraffinembedded (FFPE) NSCLC tissues (4 patients with squamous cell carcinoma and 6 adenocarcinoma) and their corresponding non-tumorous lung tissues were collected from May to October, 2012 at Subei Hospital. Another 48 FFPE NSCLC tissues were obtained from February, 2008 to December, 2009. On enrollment, tissue samples used in this study were collected prior to treatment with chemoradiotherapy. Each sample was confirmed by histopathologic evaluation through hematoxylin and eosin (H\&E) staining. Clinical data were extracted at the time of resection, and patients who entered into the registry were prospectively followed up for the ascertainment of vital status through April 30, 2012.
Cell culture. Human lung cancer A549 and H1299 cells and normal bronchial epithelial BEAS-2B cells were purchased from the Cell Resource Center, Shanghai Institute of Biochemistry. BEAS-2B cells were derived by transforming human bronchial epithelial cells with an adenovirus 12-simian virus 40 construct (23). The cell lines were maintained at $37^{\circ} \mathrm{C}$ in a humidified air atmosphere containing $5 \%$ carbon dioxide in RPMI-1640 (Wisent, Quebec, Canada) supplemented with $10 \%$ fetal bovine serum (Wisent).

RNA isolation. For cultured cells, total RNA was extracted using TRIzol reagent (Invitrogen Life Technologies, Carlsbad, CA, USA). Total tissue RNA was extracted from FFPE tissue sections using the miRNeasy FFPE kit (Qiagen, Valencia, CA, USA) according to the manufacturer's instructions. Briefly, all paraffin was removed from freshly cut FFPE tissue sections by treating with deparaffinization solution. Samples were under protease digestion to release RNA from the sections and short incubation to reverse formalin crosslinking of the released nucleic acids. DNA was removed from the RNA sample by a final DNase digestion step. Total RNA including miRNA was eventually dissolved in $20 \mu \mathrm{l}$ of RNase-free water. The concentration of RNA was measured using a NanoDrop-1000 (Thermo Fisher Scientific, Waltham, MA, USA), and RNA integrity was determined by $1.5 \%$ agarose gel electrophoresis.

Expression analysis of miRNA-148 a by quantitative real-time polymerase chain reaction ( $q R T-P C R)$. cDNA synthesis for miRNA-148a validation was performed according to the manufacturer's recommendation. Briefly, RNA was reversetranscribed (RT) using a ReverAid First Strand cDNA kit (Thermo Fisher Scientific) in combination with a stem-loop primer for miRNA-148a. Under our experimental conditions, we used U6 snRNA as an internal control for normalizing the expression levels of miRNA-148a. The RT primers were designed as follows: miRNA-148a, 5'-GTCGTATCCAG TGCAGGGTCCGAGGTATTCGCACTGGATACGACACA AAG-3' and U6, 5'-TGGTGTCGTGGAGTCG-3'. In addition, $1.0 \mu \mathrm{g}$ total RNA was combined with $4.0 \mu \mathrm{l}$ reaction buffer $(5 \mathrm{X})$, $1.0 \mu \mathrm{l}$ RiboLock RNase inhibitor, $2.0 \mu \mathrm{l}$ dNTP mix $(10 \mathrm{mM}$ each), $1.0 \mu 1$ ReverAid M-MuLV reverse transcriptase in a total reaction volume of $20 \mu \mathrm{l}$. Reactions were carried out using the ABI Prism 7900HT Fast Real-Time PCR system (Applied Biosystems, Foster City, CA, USA) using the following conditions: $25^{\circ} \mathrm{C}$ for $5 \mathrm{~min}, 42^{\circ} \mathrm{C}$ for $60 \mathrm{~min}, 70^{\circ} \mathrm{C}$ for $5 \mathrm{~min}$, and then hold at $4^{\circ} \mathrm{C}$. After the RT reaction, miRNA expression was detected using LightCycler ${ }^{\circledR} 480$ SYBR-Green I Master on the LightCycler $^{\circledR} 480$ Real-Time PCR system (both from Roche Applied Science, Indianapolis, IN, USA). cDNA products were diluted at $20 \mathrm{X}$, and the $10-\mu \mathrm{l}$ PCR mixture contained $1 \mu \mathrm{l}$ diluted RT product, $5 \mu \mathrm{l}$ SYBR-Green Master Mix, $2 \mu 1$ RNasefree water, $1 \mu \mathrm{l}$ forward, and $1 \mu \mathrm{l}$ reverse primers. The PCR primers for miRNA-148a and U6 were designed as follows: miRNA-148a forward, 5'-TCAGTGCACTACAGAACTT TGT-3' and reverse, 5'-GCTGTCAACGATACGCTACGT-3'; U6 forward, 5'-CTCGCTTCGGCAGCACA-3' and reverse, 5'-AACGCTTCACGAATTTGCGT-3'. The reaction was incubated at $95^{\circ} \mathrm{C}$ for $10 \mathrm{~min}$ followed by 40 cycles of $95^{\circ} \mathrm{C}$ for $15 \mathrm{sec}$ and $65^{\circ} \mathrm{C}$ for $60 \mathrm{sec}$. The relative miRNA-148a 
expression was calculated using the $2^{-\Delta \mathrm{Ct}}$ method, where $\Delta \mathrm{Ct}$ is the difference in threshold cycles $(\mathrm{Ct})$ for the target and reference $=\mathrm{Ct}$ (miRNA-148a) $-\mathrm{Ct}(\mathrm{U} 6)$. The RT and PCR primers were synthesized by Shenggong Biotech Co., Ltd. (Shanghai, China).

DNA isolation and bisulfite reaction. Total tissue DNA was extracted from FFPE tissue scrolls using a Qiagen EpiTect Plus FFPE Bisulfite kit (Qiagen) according to the manufacturer's instructions. Briefly, FFPE tissue scrolls were deparaffinized, followed by proteinase digestion and decrosslinking. The DNA bisulfite reaction was then set up and performed using the ABI Prism 7900HT Fast Real-Time PCR system. Upon completion of the bisulfite conversion, modified DNA was purified by several steps of clean-up and eventually eluted. The modified DNA concentration was measured using a NanoDrop-1000 and stored at $-20^{\circ} \mathrm{C}$ for further analysis.

Methylation analysis of the DNA region encoding miRNA-148a in tissue samples by quantitative methylation-specific polymerase chain reaction ( $q M S-P C R)$. As previously described, the methylation rate of miRNA-148a was determined by qMS-PCR (24). We subjected 10 ng of the modified DNA to PCR amplification with ABI Prism 7900HT Fast Real-Time PCR system and primers: miRNA-148a forward (5-TGGGTA TTTGTTTTTGTTGATTG-3) and reverse (5-ACTACACTTA AACCCCCTCTAACC-3). Reactions were carried out using the following conditions: $90^{\circ} \mathrm{C}$ for $5 \mathrm{~min}, 40$ cycles of $95^{\circ} \mathrm{C}$ for $15 \mathrm{sec}, 60^{\circ} \mathrm{C}$ for $30 \mathrm{sec}$ and $72^{\circ} \mathrm{C}$ for $15 \mathrm{sec}$; final extension of $10 \mathrm{~min}$ at $72^{\circ} \mathrm{C}$. The PCR products were diluted into water at $500 \mathrm{X}$, and $1 \mu \mathrm{l}$ diluted products was then subjected to qMS-PCR with LightCycler ${ }^{\circledR} 480$ SYBR-Green I Master. The RT primers were designed as follows: unmethylated-specific miRNA-148a forward, 5-TATGATTTGTTTTATTATTGG TT-3 and reverse, 5-AACACTAACAACATCAACAACC-3; methylated-specific miRNA-148a forward, 5-TGATTCGTTT TATTATCGGTC-3 and reverse, 5-AACACTAACGACATC GACG-3. The reaction was conducted at $95^{\circ} \mathrm{C}$ for $10 \mathrm{~min}$ followed by 40 cycles of $95^{\circ} \mathrm{C}$ for $15 \mathrm{sec}$ and $60^{\circ} \mathrm{C}$ for $60 \mathrm{sec}$ in the LightCycler ${ }^{\circledR} 480$ Real-Time PCR system. The methylation level was calculated with the formula $2^{-\Delta \mathrm{Ct}}$, where $\Delta \mathrm{Ct}$ is the difference in the threshold cycle values for methylated and unmethylated $=\mathrm{Ct}$ (methylated) $-\mathrm{Ct}$ (unmethylated). The methylation level was expressed as a percentage.

Treatment with 5-aza-2'-deoxycytidine in A549 and H1299 cells. For treatment with DNA methylation inhibitor 5-aza2'-deoxycytidine (5-aza-dC; Sigma, St. Louis, MO, USA), A549 and H1299 cells were seeded into 24-well plates on day 0 and exposed to 5 -aza-dC at a final concentration of $5 \mu \mathrm{mol} / 1$ from day 1 to 3 and then harvested for qRT-PCR assay of miRNA-148a expression on day 4.

Cell transfection. miRNA-148a mimics and negative control oligonucleotides were synthesized by GenePharma Co., Ltd. (Shanghai, China). Cell transfection was performed using Lipofectamine 2000 reagents (Invitrogen Life Technologies) according to the manufacturer's protocol. Twenty-four hours after transfection, cells were collected for qRT-PCR analysis and functional assay.
Wound-healing assay. miRNA-148a mimic-transfected cells were grown to confluence in a $24-$ well plate and wounded by dragging a $200-\mu 1$ pipette tip through the monolayer. Cells were then washed to remove cellular debris and allowed to migrate for $24 \mathrm{~h}$. Images were captured at 0 and $24 \mathrm{~h}$ after wounding under an Eclipse Ti-U (Nikon, Kanagawa, Japan) inverted microscope. The relative surface traveled by the leading edge was assessed using Image-Pro Plus version 6.0 software. Three independent experiments were performed.

Cell migration and invasion assays. For the migration assays, $2.5 \times 10^{4}$ cells were added into the upper chamber of the insert ( $8-\mu \mathrm{m}$ pore size; Corning Incorporated, Corning, NY, USA). For the invasion assays, cells were added into the upper chamber of the insert pre-coated with Matrigel (BD Biosciences, Bedford, MA, USA). In both assays, cells were plated in medium without serum, and medium containing $10 \%$ fetal bovine serum in the lower chamber served as a chemoattractant. After $40 \mathrm{~h}$ of incubation, the cells that did not migrate or invade through the pores were carefully wiped off with cotton wool. The inserts were then stained with $20 \%$ methanol and $0.2 \%$ crystal violet, imaged with an Eclipse Ti-U inverted microscope, and counted by Image-Pro Plus version 6.0.

Western blotting. Seventy-two hours after transfection, cells were collected and homogenized in lysis buffer $(50 \mathrm{mM}$ Tris- $\mathrm{HCl}, \mathrm{pH} 8.0,150 \mathrm{mM} \mathrm{NaCl}, 0.1 \%$ SDS, $1 \%$ Nonidet P-40, $0.5 \%$ sodium deoxycholate, $0.02 \%$ sodium azide, $100 \mathrm{mg} / \mathrm{l}$ PMSF, $1 \mathrm{mg} / 1$ aprotinin) and centrifuged at 11,000 x $\mathrm{g}$ for $15 \mathrm{~min}$. The supernatant was collected and the protein content was determined by BCA protein reagent (Pierce Chemical Co., Rockford, IL, USA). The proteins (50 $\mu \mathrm{g} /$ lane) were separated on $10 \%$ SDS-PAGE gels and transferred to PVDF membranes. Membranes were blocked with $5 \%$ nonfat milk and incubated with primary antibodies against DNMT1 (Abcam, Cambridge, MA, USA), E-cadherin and $\beta$-actin (Santa Cruz Biotechnology, Inc., Santa Cruz, CA, USA) overnight at $4^{\circ} \mathrm{C}$. After washing, the membranes were subjected to continued incubation with goat anti-rabbit or anti-mouse secondary antibody (Santa Cruz Biotechnology, Inc.) and visualized with enhanced chemiluminescence.

Methylation-specific PCR (MSP) for promoters of E-cadherin Genomic DNA was obtained from the cell lines and modified with sodium bisulfite as described above. The modified DNAs were specifically amplified by methylated and unmethylated primers of E-cadherin as previously described (25). The PCR products were separated by high-resolution $4 \%$ agarose E-gels (Invitrogen Life Technologies) and visualized under UV illumination.

Statistical analysis. The results were analyzed using the SPSS 13.0 statistical software. Data are shown as means \pm standard deviation (SD), and the Student's t-test was used for statistical analysis. Correlation between miRNA-148a expression and the methylation level of the DNA region encoding miRNA-148a in tissues was evaluated by Pearson's correlation. Descriptive statistics for the study population were generated by miRNA-148a expression for demographic and clinicopathologic characteristics. Categorical variables were 

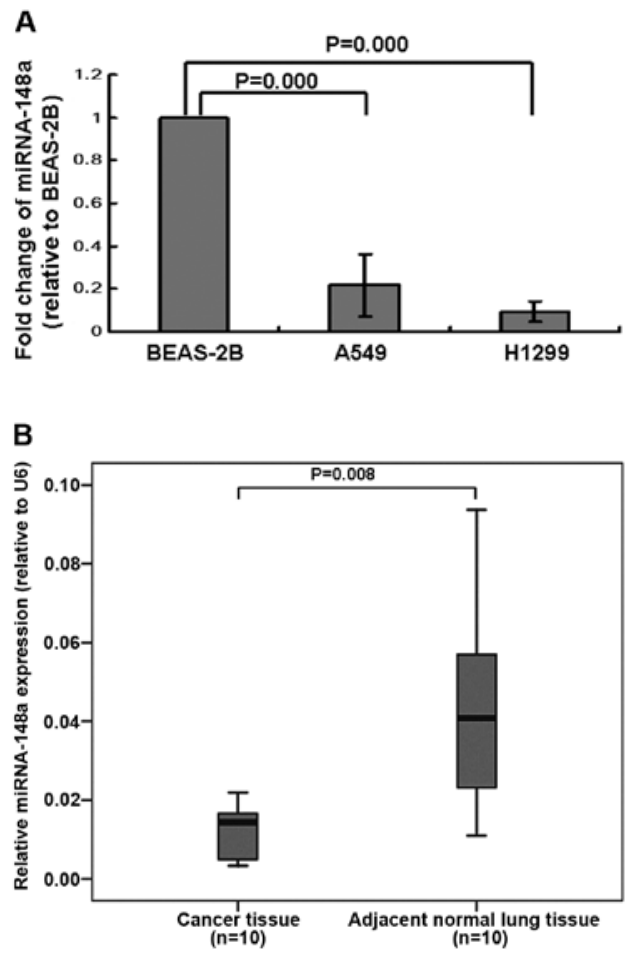

Figure 1. Downregulation of miRNA-148a expression in lung cancer cells and tumors. (A) Relative expression levels of miRNA-148a in lung adenocarcinoma A549 and H1299 cells and BEAS-2B normal bronchial epithelial cells were determined by qRT-PCR assay, and normalized to U6 levels using the $2^{-\Delta \mathrm{Ct}}$ method. Values were reported as fold-change relative to BEAS-2B cells. Results represent the means \pm SD from 3 independent experiments. (B) Relative expression of miRNA-148a in 10 pairs of lung cancer tissue and their corresponding non-tumorous lung tissue was determined by qRT-PCR assay, and normalized to U6 levels.

compared using analysis of variance test. Comparing factors for miRNA-148a expression included gender, age, pathology, smoking status, tumor size, histologic grade, T stage, lymph node metastasis and clinical stage. Univariate Kaplan-Meier method was performed to estimate disease-free survival and overall survival according to miRNA-148a expression. Survival differences according to expression were analyzed using the log-rank test. For the analysis of disease-free and overall survival, patients who died prior to recurrence were considered censored at death. Multivariable Cox proportional hazards model analysis of factors potentially related to survival was used to identify the significant influence of miRNA-148a expression on survival, adjusted for gender, age, histologic grade, $\mathrm{T}$ stage and smoking status. $\mathrm{P}<0.05$ was considered to indicate a statistically significant result.

\section{Results}

miRNA-148a is downregulated in lung cancer cell lines and tumor tissues. The expression levels of miRNA-148a in A549 and H1299 lung cancer cells and in BEAS-2B normal lung bronchial epithelial cells were determined by qRT-PCR. Our results showed that the levels of miRNA-148a were dramatically downregulated in lung cancer A549 and H1299 cells when compared with BEAS-2B cells (Fig. 1A). Next, we analyzed the expression levels of miRNA-148a in 10 pairs of FFPE lung cancer tissue specimens and matched adjacent

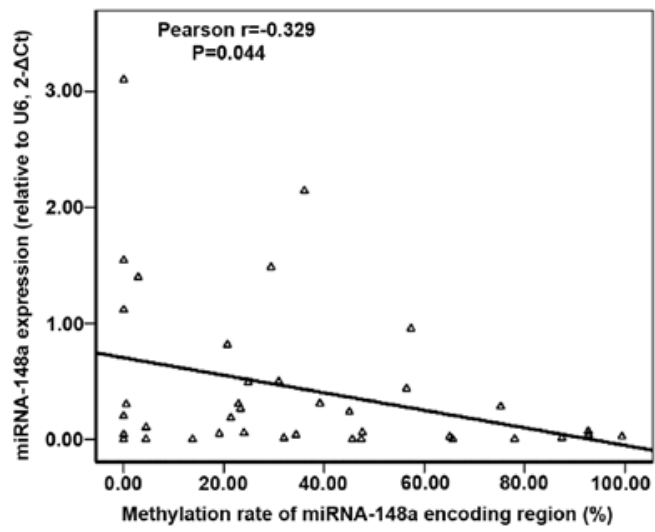

Figure 2. Correlation of expression of miRNA-148a and the methylation level of the DNA region encoding miRNA-148a in tissue samples of NSCLC patients. An inverse correlation was found between the expression of miRNA-148a and the methylation rate of the miRNA-148a encoding region evaluated by Pearson's regression in lung cancer tissues (Pearson $r=-0.329$, $\mathrm{P}=0.044)$. NSCLC, non-small cell lung cancer.

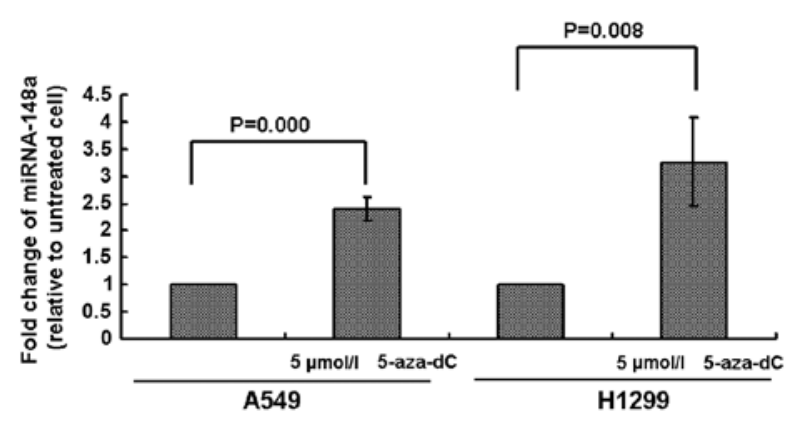

Figure 3. Fold changes in miRNA-148a expression in lung cancer cells $72 \mathrm{~h}$ after treatment with 5-aza-dC. miRNA-148a was dramatically upregulated (compared with untreated cells) following treatment with $5 \mu \mathrm{mol} / 1$ of 5-aza-dC in A549 and H1299 cells.

normal lung tissues. Consistent with the results in the cell lines, the expression levels of miRNA-148a in lung cancer tissues were significantly decreased when compared to that in the matched normal lung tissues (Fig. 1B).

Hypermethylation of the miRNA-148a gene results in the silencing of miRNA-148a in lung cancer. There are many factors that reduce the expression of miRNAs. CpG plot software (http://bioweb.pasteur.fr) reported two $\mathrm{CpG}$ islands located close to the miRNA-148a gene, and the hypermethylation of the DNA region encoding miRNA-148a was reported to be responsible for its repression (24). We retrospectively tested miRNA-148a expression in 48 FFPE lung cancer tissues using qRT-PCR. We also analyzed the methylation status of the DNA region encoding miRNA-148a in the same population by means of qMS-PCR assay and found that 38 tumor tissue samples were available for further analysis. Among the analyzed tissues, we found an inverse correlation between the expression level of miRNA-148a and the methylation rates of the miRNA148a encoding region in lung cancer tissues as evaluated by Pearson's regression $(\mathrm{P}=0.044$, Fig. 2). The correlation coefficient was -0.329 . To further identify whether miRNA-148a was aberrantly inhibited by DNA hypermethylation, we treated 
Table I. Associations between the expression of miRNA-148a and clinicopathologic features in patients with NSCLC.

\begin{tabular}{|c|c|c|c|c|}
\hline Characteristics & No. of patients & Relative miRNA-148a expression $\left(2^{-\Delta \mathrm{Ct}}\right)$ & F-value & P-value \\
\hline \multicolumn{5}{|l|}{ Gender } \\
\hline Male & 37 & $0.5922 \pm 0.7646$ & 2.346 & 0.132 \\
\hline Female & 11 & $0.2227 \pm 0.4066$ & & \\
\hline \multicolumn{5}{|l|}{ Age (years) } \\
\hline$\leq 60$ & 21 & $0.5849 \pm 0.8000$ & 0.435 & 0.513 \\
\hline$>60$ & 27 & $0.4473 \pm 0.6454$ & & \\
\hline \multicolumn{5}{|l|}{ Pathology } \\
\hline Squamous & 25 & $0.6437 \pm 0.8492$ & 1.946 & 0.170 \\
\hline Adenocarcinoma & 23 & $0.3595 \pm 0.5036$ & & \\
\hline \multicolumn{5}{|l|}{ Smoking status } \\
\hline Non-smoker & 20 & $0.2941 \pm 0.4823$ & 3.222 & 0.079 \\
\hline Smoker & 28 & $0.6599 \pm 0.8136$ & & \\
\hline \multicolumn{5}{|l|}{ Tumor size (cm) } \\
\hline$\leq 4$ & 34 & $0.6289 \pm 0.7964$ & 3.568 & 0.065 \\
\hline$>4$ & 14 & $0.2128 \pm 0.3045$ & & \\
\hline \multicolumn{5}{|l|}{ Histologic grade } \\
\hline I & 12 & $0.5404 \pm 0.4651$ & 0.033 & 0.856 \\
\hline II-III & 36 & $0.4965 \pm 0.7830$ & & \\
\hline \multicolumn{5}{|l|}{ pT stage } \\
\hline $\mathrm{T} 1$ & 25 & $0.5904 \pm 0.7226$ & 0.701 & 0.407 \\
\hline $\mathrm{T} 2+\mathrm{T} 3$ & 23 & $0.4174 \pm 0.7060$ & & \\
\hline \multicolumn{5}{|c|}{ Lymph node metastasis } \\
\hline Negative & 26 & $0.6977 \pm 0.8781$ & 4.329 & 0.043 \\
\hline Positive & 22 & $0.2827 \pm 0.3468$ & & \\
\hline \multicolumn{5}{|l|}{ pTNM stage } \\
\hline I & 20 & $0.8437 \pm 0.9377$ & 4.387 & 0.018 \\
\hline II & 17 & $0.2876 \pm 0.3205$ & & \\
\hline III & 11 & $0.2361 \pm 0.4015$ & & \\
\hline
\end{tabular}

NSCLC, non-small cell lung cancer.

A549 and H1299 cells with $5 \mu \mathrm{mol} / 15$-aza-dC for $72 \mathrm{~h}$ and then analyzed the expression of miRNA-148a. As shown in Fig. 3 , the expression of miRNA-148a was increased in both A549 and H1299 cells, suggesting that DNA methylation was involved in the silencing of miRNA-148a in lung cancer.

Decreased expression of miRNA-148a is associated with lymph node metastasis and advanced clinical stage. To determine whether miRNA-148a expression is associated with clinicopathologic features of lung cancer, we further analyzed the relationship between miRNA-148a expression and clinicopathologic characteristics of lung cancer. We found that patients with lymph node metastasis and advanced clinical stage had a significantly lower expression of miRNA-148a $(\mathrm{P}=0.043$ and 0.018 respectively, Table $\mathrm{I})$. There were no significant differences between expression of miRNA-148a and other clinicopathologic characteristics of the lung cancer cases including gender, age, pathology, smoking status, tumor size, histologic grade and T stage (Table I).
Low levels of miRNA-148a expression are associated with shortened survival. To further evaluate the correlation between miRNA-148a expression and the prognosis of NSCLC patients, Kaplan-Meier survival analysis and multivariable Cox proportional hazards analysis were performed. In this study, one patient died from pneumonia shortly after surgery. Thus, according to the median relative miRNA-148a expression (0.2638) in the remaining 47 tumor tissues, patients were classified into 2 groups: the low miRNA-148a expression group $(n=24)$ with miRNA-148a expression less than or equal to the median; and the high miRNA-148a group $(n=23)$, with miRNA-148a expression greater than the median. In the univariate Kaplan-Meier analysis, patients with low expression of miRNA-148a had a significantly shorter disease-free survival $(\mathrm{P}=0.030$, Fig. $4 \mathrm{~A})$ and overall survival $(\mathrm{P}=0.006$, Fig. 4B) when compared with the patients with high expression of miRNA-148a. In the multivariable Cox proportional hazards analysis, adjusting for gender, age, histologic grade, smoking status and $\mathrm{T}$ stage, our results showed that patients with low 
A

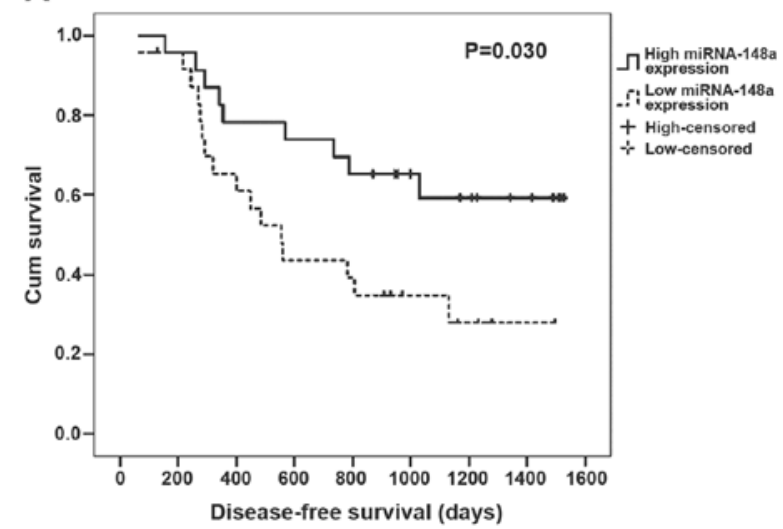

B

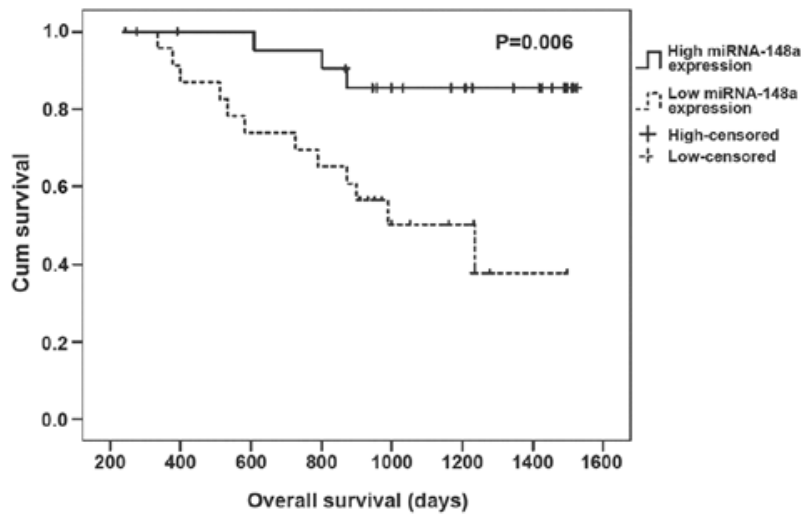

Figure 4. Kaplan-Meier survival curves for NSCLC patients with miRNA-148a-high and miRNA-148a-low expression. (A) Kaplan-Meier estimate of diseasefree survival for NSCLC patients according to miRNA-148a expression. (B) Kaplan-Meier estimate of overall survival for NSCLC patients according to miRNA-148a expression. The survival data were compared using the log-rank test. Patients with low miRNA-148a expression showed shorter disease-free and overall survival than patients with high miRNA-148a expression. NSCLC, non-small cell lung cancer.

Table II. Cox proportional hazards model analysis of adjusted hazard ratios for disease-free and overall survival according to miRNA-148a expression in NSCLC patients.

\begin{tabular}{llcl}
\hline $\begin{array}{l}\text { miRNA-148a } \\
\text { expression }\end{array}$ & HR & $\begin{array}{c}\text { 95\% CI } \\
\text { (lower-upper) }\end{array}$ & P-value \\
\hline
\end{tabular}

\begin{tabular}{llll}
\hline Disease-free survival & & & 0.178 \\
High & 1.000 & & \\
Low & 1.927 & $0.742-5.006$ & \\
Overall survival & & & 0.022 \\
High & 1.000 & & \\
Low & 5.018 & $1.257-20.028$ & \\
\hline
\end{tabular}

NSCLC, non-small cell lung cancer; HR, hazard ratio; CI, confidence interval.

expression of miRNA-148a had a higher risk of tumor-related death (hazard ratio, 5.018; 95\% CI, 1.257-20.028, $\mathrm{P}=0.022$, Table II) compared with the patients with high expression of miRNA-148a; expression of miRNA-148a was not significantly associated with disease-free survival $(\mathrm{P}=0.178)$.

miRNA-148a suppresses lung cancer A549 and H1299 cell invasion in vitro. Since we observed that the downregulation of miRNA-148a in lung cancer was closely associated with lymph node metastasis and poor survival, we postulated that overexpression of miRNA-148a in lung cancer cells may exert inhibitory effects on cell invasion and metastasis. We measured the difference in the migratory capability of lung cancer A549 and H1299 cells using transient transfection. The wound-healing assay showed that miRNA-148a mimic-transfected A549 and H1299 cells had a significantly lower capability of migration than the negative control cells (Fig. 5A). Moreover, overexpression of miRNA-148a significantly suppressed the migratory and invasive abilities of cells as determined by Transwell migration and Matrigel invasion assays (Fig. 5B). These results strongly suggest that miRNA-148a downregulation was associated with high migration capability of lung cancer cells.

miRNA-148a downregulates DNMT1 expression in lung cancer cells. It was predicted that both the 3'UTR and the gene coding DNA sequence (CDS) of DNMT1 had putative miRNA-148a binding elements, and miRNA-148a was found to inhibit DNMT1 protein expression in $\mathrm{CD}^{+} \mathrm{T}$ cells and gastric cancer cells $(19,26)$. To explore the molecular mechanism of miRNA-148a in lung cancer metastasis, we examined whether the expression of DNMT1 was regulated by miRNA148a in lung cancer cells. As shown in Fig. 6A, our results showed that the protein levels of DNMT1 were significantly inhibited in both A549 and H1299 cells after transfection with the miRNA-148a mimics.

miRNA-148a induces the overexpression of methylation-sensitive gene E-cadherin in lung cancer cells. In lung cancer, a number of methylation-sensitive genes including E-cadherin that are linked to lung cancer metastasis are downregulated, and E-cadherin was found to be demethylated and overexpressed following treatment with the DNMT inhibitor in lung cancer cells (25). As described above, miRNA-148a inhibited DNMT1 protein expression in lung cancer cells. We next examined whether miRNA-148a induces the overexpression of E-cadherin. Results showed that the protein levels of E-cadherin were significantly upregulated in A549 and H1299 cells after transfection with miRNA-148a mimics (Fig. 6A). We further used MSP to determine the methylation status of the E-cadherin promoter post-transfection of miRNA-148a mimics in lung cancer cells. Consistent with a previous study (25), the results showed that the A549 and H1299 cells had a nearly complete methylation of the E-cadherin promoter (Fig. 6B). We also showed that restoration of E-cadherin was significantly associated with its promoter demethylation in the A549 and H1299 cell lines (Fig. 6B). Taken together, our results suggest that the inhibition of migration by miRNA-148a may be associated with DNMT1-mediated epigenetic regulation of E-cadherin. 
A
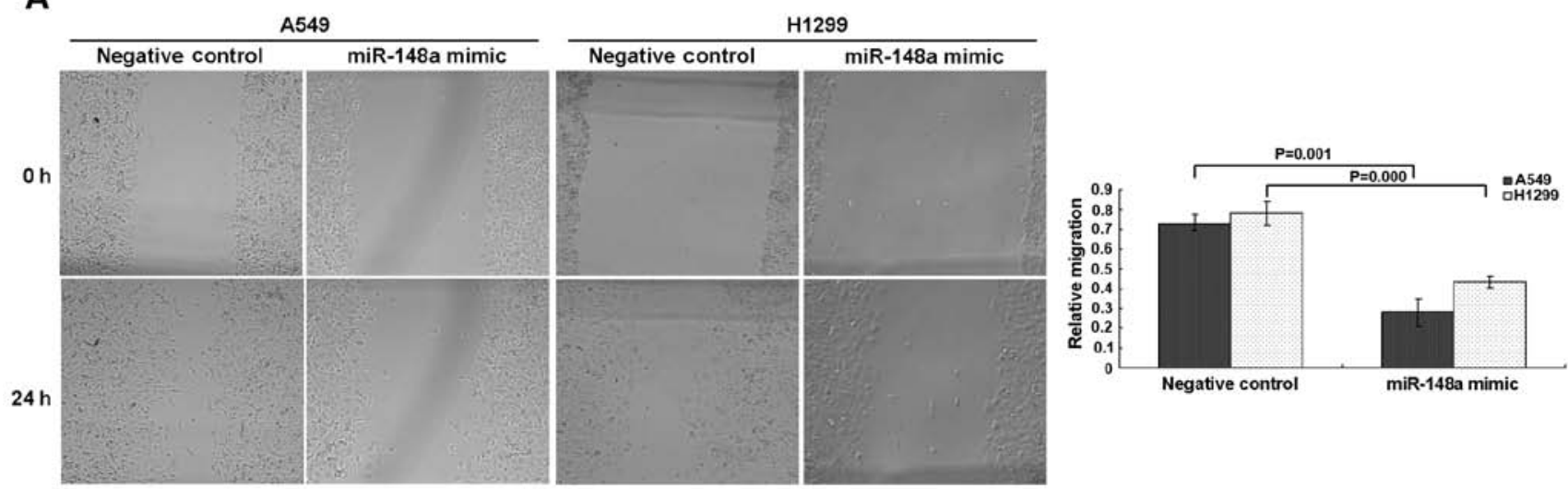

B
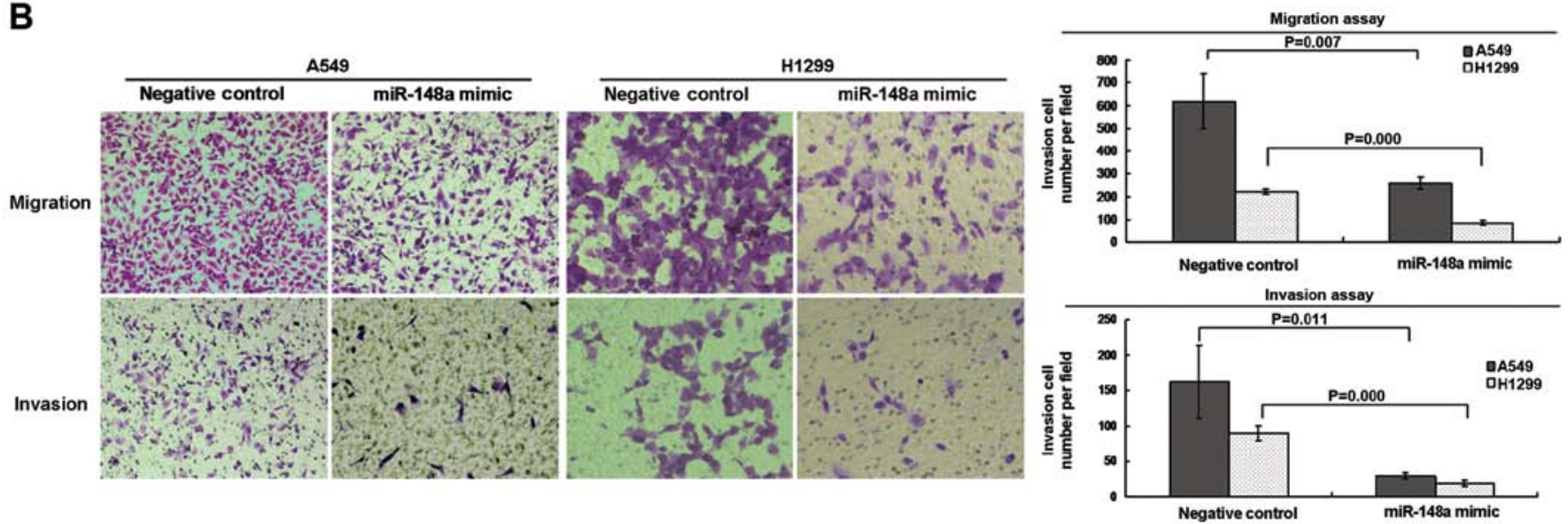

Figure 5. miRNA-148a suppresses lung cancer cell migration and invasion. (A) Wound-healing assays. Illustrative examples of the wound-healing assays in the negative control and miRNA-148a mimic-transfected lung cancer cell lines at 0 and $24 \mathrm{~h}$. miRNA-148a mimic-transfected cells exhibited minor invasion capability. (B) Transwell assays. Illustrative examples of Transwell migration and Matrigel invasion assays in the negative control and miRNA-148a mimic-transfected cell lines at 0 and $40 \mathrm{~h}$. Overexpression of miRNA-148a significantly impeded the cell migratory and invasive abilities in the A549 and H1299 cells.

\section{Discussion}

Studies have revealed that miRNAs constitute a robust and complicated regulatory network by post-transcriptional regulation of almost one third of human genes. Aberrant expression of miRNAs has been reported in several tumor types and may play a critical role in carcinogenesis and tumor progression. Furthermore, dysregulation of miRNA expression has also been correlated with outcome or prognosis in many cancer types (10). Currently, miRNAs are able to be successfully detected in many sample types, including fresh frozen tissue samples, FFPE and blood samples. Published comparisons from research using fresh frozen tissue samples versus FFPE for qRT-PCR and microarray analysis have demonstrated a good correlation for miRNA expression profiles derived from both sample types. Since FFPE samples are easily stored in hospitals and remain stable for many years, FFPE samples are believed to provide a feasible alternative to fresh frozen tissue samples for miRNA expression analysis $(9,27-29)$. In the present study, we used FFPE samples to detect the expression of miRNA-148a in NSCLC. Our qRT-PCR results showed that the expression of miRNA-148a was successfully detected in NSCLC FFPE samples stored in our hospital, which enabled further evaluation of the clinical value of miRNA-148a expression in NSCLC.

Recently, Watanabe et al (11) assessed the expression of miRNA-148a in primary tumor samples and found that expression of miRNA-148a was lower when compared to that in normal lung tissue. Since miRNA-148a is widely accepted to be an miRNA with tumor-suppressor activity in different types of cancer (20-22), there are only limited data available concerning the role of miRNA-148a in NSCLC. In our study, the miRNA-148a expression data were gathered from 10 pairs of FFPE tissue samples and 3 cell lines (A549, H1299 and BEAS-2B cells). The results demonstrated a significant difference in expression between tumor and normal samples.

There are many factors that reduce the expression of miRNAs. DNA methylation of $\mathrm{CpG}$ islands is one of the important regulatory mechanisms for gene expression, which has also been found to be responsible for inactivating miRNA expression including miRNA-148a (19). In a previous study, miRNA-148a was found to be silenced by specific hypermethylation in many types of cancers when compared with normal tissues. The involvement of miRNA-148a hypermethylation was also frequently observed in human primary malignancies such as colon, breast, head and neck carcinomas and 
A
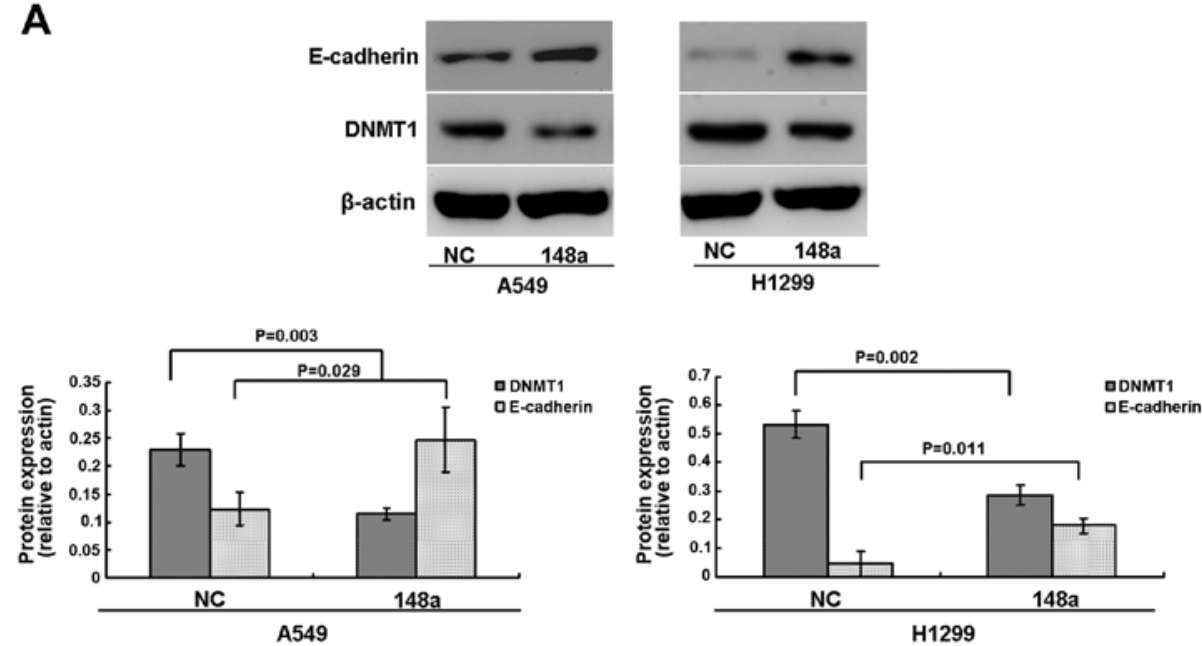

B
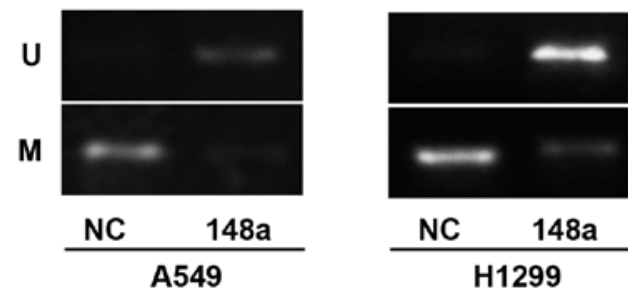

Figure 6. miRNA-148a-mediated DNMT1 depletion induces E-cadherin expression via demethylation of the E-cadherin promoter in A549 and H1299 cells. (A) Transfection with miRNA-148a mimics (148a) led to a decrease in the level of DNMT1 and an increase in E-cadherin in both lung cancer cell lines when compared with the negative control (NC). DNMT1, E-cadherin and $\beta$-actin levels were measured by western blotting and normalized by actin. (B) Methylation status of the promoter was analyzed using MSP post-transfection with the miRNA-148a mimics or the negative control. miRNA-148a demethylated the E-cadherin promoter in the A549 and H1299 cells. U, unmethylation reaction; M, methylation reaction. DNMT1, DNA methyltransferase 1.

melanomas (21). In addition the aberrant hypermethylation of the miRNA-148a coding region may lead to downregulation of miRNA-148a expression in pancreatic ductal adenocarcinomas (24). We examined retrospectively miRNA-148a expression in another 48 FFPE lung cancer tissues, and our present study showed that low expression of miRNA-148a was associated with a high methylation level of the miRNA-148a coding region in lung cancer tissues. Intriguingly, miRNA148a was upregulated by the treatment of the DNMT inhibitor 5-aza-dC in A549 and H1299 cells, which suggest that methylation of miRNA-148a may be one possible mechanism which leads to silencing of miRNA-148a in human lung cancer.

Currently, the association of altered miRNA expression and cancerogenesis, tumor progression as well as patient survival is well established $(7,30,31)$. In patients with esophageal squamous cell cancer, Hummel et al (9) found a significant negative association between miRNA-148a expression and the risk of tumor recurrence and tumor-related death. In our study, we found a correlation between decreased expression of miRNA-148a and clinical signs of a more aggressive and advanced tumor stage in NSCLC patients. Our present study also showed that low miRNA-148a expression was significantly associated with a shortened disease-free and overall patient survival. Furthermore, multivariable Cox proportional hazards analysis also indicated that the expression of miRNA148a was an independent prognostic factor for overall survival.

To further determine the role of miRNA-148a in tumor progression and metastatic behavior in lung cancer A549 and
H1299 cell lines, wound-healing and Transwell migration assays were carried out. Our functional studies showed that overexpression of miRNA-148a suppressed cell migration and invasion in vitro. Similar to our results, Lujambio et al (21) found that the reintroduction of miRNA-148a in SIHN-011B (head and neck cancer) cancer cells inhibited tumor motility and metastasis in vitro and in vivo. Moreover, miRNA-148a was also reported to function as a tumor metastasis suppressor to suppress cancer cell migration, invasion and lung metastasis in gastric cancer (22). Taken together, our results suggest that miRNA-148a functions as a negative regulator for lung cancer metastasis.

In our study, the mechanism by which miRNA-148a inhibited the migratory and invasive abilities of NSCLC cells was further investigated. We found that levels of DNMT1 were significantly inhibited by miRNA-148a in lung cancer cells. Supporting our result, DNMT1 was recently confirmed as a direct target of miRNA-148a $(19,26)$. DNMT1 is essential for maintaining DNA methylation patterns of cells, which causes inactivation of methylation-sensitive genes including metastatic repressor gene E-cadherin, while the depletion of DNMT1 is sufficient to result in gene overexpression via promoter demethylation $(25,32,33)$. There is increasing evidence to support the correlation between reduced E-cadherin expression and gene promoter methylation in lung cancer $(25,34)$. Moreover the loss of E-cadherin function was found in several invasive cancers including lung cancer and was associated with metastasis and invasion. The present study showed that 
enforced miRNA-148a expression restored E-cadherin gene expression by demethylating its promoter. Similar to our results, inhibition of miRNA-152 increased global DNA methylation in liver cell lines including the methylation levels of E-cadherin by targeting DNMT1 (33). Hence, we speculated that the inhibition of DNMT1 induced by miRNA-148a leads to hypomethylation of E-cadherin, which results in re-expression of E-cadherin in lung cancer cells. This in turn leads to inhibition of cell invasion in human lung cancers.

In conclusion, we describe miRNA-148a as an important anti-metastatic miRNA which was downregulated in NSCLC. Downregulated levels of miRNA-148a due to DNA methylation were correlated with aggressive tumor behavior and poor prognosis in patients with NSCLC. Our study also provides an important rationale for developing epigenetic therapies targeting miRNA-148a to re-express the methylation-silenced tumor-suppressor gene E-cadherin in lung cancer. miRNA-148a may have the potential to assist with preoperative assessment and provide additional information to complement clinical decisions.

\section{References}

1. Jemal A, Tiwari RC, Murray T, et al: Cancer statistics, 2004. CA Cancer J Clin 54: 8-29, 2004.

2. Minna JD, Roth JA and Gazdar AF: Focus on lung cancer. Cancer Cell 1: 49-52, 2002.

3. Dykxhoorn DM: MicroRNAs and metastasis: little RNAs go a long way. Cancer Res 70: 6401-6406, 2010.

4. Yanaihara N, Caplen N, Bowman E, et al: Unique microRNA molecular profiles in lung cancer diagnosis and prognosis Cancer Cell 9: 189-198, 2006.

5. Farazi TA, Spitzer JI, Morozov $P$ and Tuschl T: miRNAs in human cancer. J Pathol 223: 102-115, 2011.

6. Garzon R, Fabbri M, Cimmino A, Calin GA and Croce CM: MicroRNA expression and function in cancer. Trends Mol Med 12: 580-587, 2006.

7. Zhang B, Pan X, Cobb GP and Anderson TA: microRNAs as oncogenes and tumor suppressors. Dev Biol 302: 1-12, 2007.

8. Schetter AJ, Leung SY, Sohn JJ, et al: MicroRNA expression profiles associated with prognosis and therapeutic outcome in colon adenocarcinoma. JAMA 299: 425-436, 2008.

9. Hummel R, Hussey DJ, Michael MZ, et al: MiRNAs and their association with locoregional staging and survival following surgery for esophageal carcinoma. Ann Surg Oncol 18: 253-260, 2011.

10. Langevin SM, Stone RA, Bunker CH, et al: MicroRNA-137 promoter methylation is associated with poorer overall survival in patients with squamous cell carcinoma of the head and neck. Cancer 117: 1454-1462, 2011.

11. Watanabe K, Emoto N, Hamano E, et al: Genome structurebased screening identified epigenetically silenced microRNA associated with invasiveness in non-small-cell lung cancer. Int J Cancer 130: 2580-2590, 2012.

12. Visone R, Rassenti LZ, Veronese A, et al: Karyotype-specific microRNA signature in chronic lymphocytic leukemia. Blood 114: 3872-3879, 2009.

13. Magrelli A, Azzalin G, Salvatore M, et al: Altered microRNA expression patterns in hepatoblastoma patients. Transl Oncol 2: $157-163,2009$.

14. Xu Q, Jiang Y, Yin Y, et al: A regulatory circuit of miR-148a/152 and DNMT1 in modulating cell transformation and tumor angiogenesis through IGF-IR and IRS1. J Mol Cell Biol 5: 3-13, 2013.
15. Ueda T, Volinia S, Okumura $\mathrm{H}$, et al: Relation between microRNA expression and progression and prognosis of gastric cancer: a microRNA expression analysis. Lancet Oncol 11: 136-146, 2010

16. Katada $\mathrm{T}$, Ishiguro $\mathrm{H}, \mathrm{Kuwabara} \mathrm{Y}$, et al: microRNA expression profile in undifferentiated gastric cancer. Int J Oncol 34: 537-542, 2009.

17. Chen Y, Song Y, Wang Z, et al: Altered expression of MiR-148a and MiR-152 in gastrointestinal cancers and its clinical significance. J Gastrointest Surg 14: 1170-1179, 2010.

18. Zhang H, Li Y, Huang Q, et al: MiR-148a promotes apoptosis by targeting Bcl-2 in colorectal cancer. Cell Death Differ 18: 1702-1710, 2011

19. Zhu A, Xia J, Zuo J, et al: MicroRNA-148a is silenced by hypermethylation and interacts with DNA methyltransferase 1 in gastric cancer. Med Oncol 29: 2701-2709, 2012.

20. Liffers ST, Munding JB, Vogt M, et al: MicroRNA-148a is down-regulated in human pancreatic ductal adenocarcinomas and regulates cell survival by targeting CDC25B. Lab Invest 91: 1472-1479, 2011.

21. Lujambio A, Calin GA, Villanueva A, et al: A microRNA DNA methylation signature for human cancer metastasis. Proc Natl Acad Sci USA 105: 13556-13561, 2008.

22. Zheng B, Liang L, Wang C, et al: MicroRNA-148a suppresses tumor cell invasion and metastasis by downregulating ROCK1 in gastric cancer. Clin Cancer Res 17: 7574-7583, 2011.

23. Reddel RR, Ke Y, Gerwin BI, et al: Transformation of human bronchial epithelial cells by infection with SV40 or adenovirus-12 SV40 hybrid virus, or transfection via strontium phosphate coprecipitation with a plasmid containing SV40 early region genes. Cancer Res 48: 1904-1909, 1988.

24. Hanoun N, Delpu Y, Suriawinata AA, et al: The silencing of microRNA 148a production by DNA hypermethylation is an early event in pancreatic carcinogenesis. Clin Chem 56: 1107-1118, 2010.

25. Wang G, Hu X, Lu C, Su C, Luo S and Luo ZW: Promoterhypermethylation associated defective expression of E-cadherin in primary non-small cell lung cancer. Lung Cancer 62: 162-172, 2008.

26. Pan W, Zhu S, Yuan M, et al: MicroRNA-21 and microRNA-148a contribute to DNA hypomethylation in lupus $\mathrm{CD} 4^{+} \mathrm{T}$ cells by directly and indirectly targeting DNA methyltransferase 1 . J Immunol 184: 6773-6781, 2010.

27. Xi Y, Nakajima G, Gavin E, et al: Systematic analysis of microRNA expression of RNA extracted from fresh frozen and formalin-fixed paraffin-embedded samples. RNA 13: 1668-1674, 2007.

28. Zhang X, Chen J, Radcliffe T, Lebrun DP, Tron VA and Feilotter H: An array-based analysis of microRNA expression comparing matched frozen and formalin-fixed paraffin-embedded human tissue samples. J Mol Diagn 10: 513-519, 2008.

29. Leite KR, Canavez JM, Reis ST, et al: miRNA analysis of prostate cancer by quantitative real time PCR: comparison between formalin-fixed paraffin embedded and fresh-frozen tissue. Urol Oncol 29: 533-537, 2011.

30. Esquela-Kerscher A and Slack FJ: Oncomirs - microRNAs with a role in cancer. Nat Rev Cancer 6: 259-269, 2006.

31. Calin GA and Croce CM: MicroRNA-cancer connection: the beginning of a new tale. Cancer Res 66: 7390-7394, 2006.

32. Qiu X, Qiao F, Su X, Zhao Z and Fan H: Epigenetic activation of E-cadherin is a candidate therapeutic target in human hepatocellular carcinoma. Exp Ther Med 1: 519-523, 2010.

33. Huang J, Wang Y, Guo Y and Sun S: Down-regulated microRNA-152 induces aberrant DNA methylation in hepatitis $B$ virus-related hepatocellular carcinoma by targeting DNA methyltransferase 1. Hepatology 52: 60-70, 2010.

34. Shimamoto T, Ohyashiki JH, Hirano T, Kato H and Ohyashiki K: Hypermethylation of E-cadherin gene is frequent and independent of $\mathrm{p} 16^{\mathrm{INK} 4 \mathrm{~A}}$ methylation in non-small cell lung cancer: Potential prognostic implication. Oncol Rep 12: 389-395, 2004. 